\title{
Sífilis materna e sífilis congênita notificadas em um hospital de ensino
}

RESUMO | Objetivo: Conhecer o perfil dos casos de sífilis materna e sífilis congênita notificados em um hospital de ensino. Método: Estudo descritivo, retrospectivo e documental, com análise quantitativa, dos casos de sífilis materna e sífilis congênita notificados pelo Núcleo de Vigilância Epidemiológica Hospitalar (NVEH) de um hospital de ensino, no período de 2007 a 2017, totalizando 114 casos. Resultados: A faixa etária predominante foi entre 20 a 24 anos, da cor branca e com o ensino fundamental incompleto, 86 parceiros não foram tratados concomitantemente com a mulher. Quanto ao sexo dos recém natos, 58 eram masculinos e 53 femininos, predominando a cor branca, 96 evoluíram com vida, 83 dos recém natos tiveram o teste não treponêmico reagente. Conclusão: A sífilis é um problema de saúde pública, embora o diagnóstico e o tratamento sejam de fácil acesso e de baixo custo, esse agravo ainda é um desafio para a redução dos casos.

Palavras-chaves: Sífilis, Sífilis Congênita, Notificação de Doenças.

ABSTRACT | Objective: Knowing the maternal syphilis profile and congenital syphilis reported in a school hospital. Methods: Descriptive, retrospective and documental study with quantitative analysis of maternal and congenital syphilis reported by a Hospital Epidemiological Surveillance Nucleus (HESN) of a school hospital from 2007 to 2017, totaling 114 cases. Results: The predominant age group were between 20 to 24 years old, of white skin color and with incomplete middle education. 86 partners were not treated at the same time with the woman. As for the sex of newborn people, 58 were male and 53 female and with white skin color, 96 evolved with life, 83 de los recién nascidos tuvieron la prueba de reactivos no treponemicos. Conclusion: Syphilis is a public health problem, although the diagnosis and the treatment are easy to access and unexpansive,this grievance is still a challenge to reduce the cases. Keywords: Syphilis, Congenital Syphilis, Disease notification.

RESUMEN I Objetivo: Conocer el perfil de los casos de sífilis materna y sífilis congénita notificados en un hospital de enseñanza. Métodos: Estudio descriptivo, retrospectivo y documental, con análisis cuantitativo, de los casos de sífilis materna y sífilis congénita notificados por el Núcleo de Vigilancia Epidemiológica Hospitalaria (NVEH) de un hospital de enseñanza, en el período de 2007 a 2017, con total de 114 casos. Resultados: La edad predominante fue entre 20 a 24 años, de color blanco y con la enseñanza fundamental incompleta. 86 parejas no fueron tratados concomitante con la mujer. En cuanto al sexo de los recién nacidos, 58 eran masculinos y 53 femeninos y de color blanco y 96 se desarrollaron con vida, 83 of the newborns had the non-treponemal reagent test. Conclusión: La sifilis es un problema de salud pública, aunque el diagnóstico y el tratamiento son de fácil acceso y de bajo costo, este agravio sigue siendo un desafío para la reducción de los casos.

Descriptores: Sífilis, Sífilis Congénita, Notificación de Enfermedades.

\section{Maristela Salete Maraschin}

Enfermeira. Mestre. Docente da Universidade Estadual do Oeste do Paraná/UNIOESTE, campus Cascavel/PR e do Programa de Residência em Enfermagem na Especialidade em Vigilância em Saúde e Controle de Infecções.

\section{Heverton Souza Beraldo}

Enfermeiro. Especialista em Unidade de Terapia Intensiva e Nefrologia. Residente em Enfermagem em Vigilância em Saúde e Controle de infecções da Universidade Estadual do Oeste do Paraná/ UNIOESTE, campus CascavelPR.

\section{Drieli Wawzeniak de Anchieta}

Enfermeira. Residente em Enfermagem em Vigilância em Saúde e Controle de infecções da Universidade Estadual do Oeste do Paraná/ UNIOESTE, campus Cascavel/PR.

\section{Bruna Taís Zack}

Enfermeira. Residente em Enfermagem em Vigilância em Saúde e Controle de infecções da Universidade Estadual do Oeste do Paraná/UNIOESTE, campus Cascavel/PR.

Recebido em: 09/05/2019

Aprovado em: 23/07/2019

\section{INTRODUÇÃO}

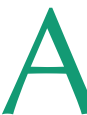
sífilis é motivo de preocupação em razão da transmissibilidade e magnitude. A doença pode acometer todas as faixas etárias e ambos os sexos e medidas de controle e prevenção dessa infecção são essenciais, tendo como base os fatores culturais e comportamentais (1). É uma infecção sistêmica, de evolução crônica, que ocupa uma importância significativa entre os problemas mais frequentes de saúde pública ${ }^{(2)}$.

No Brasil, ao ano, 50 mil parturientes têm o diagnóstico de sífilis, com prevalência variando de 1,1 a 11,5\%, em função da assistência ao pré natal e do grau de instrução materna. O resultado é que, ao ano, aproximadamente $12 \mathrm{mil}$ nascidos vivos têm sífilis congênita (SC) no Brasil ${ }^{(3)}$.

A doença é classificada em duas ca- tegorias, sendo a sífilis adquirida, a que a transmissão ocorre por meio sexual e hematogênica, sendo a forma sexual a mais comum. A sífilis congênita refere-se à doença transmitida verticalmente para feto ${ }^{(4-5)}$. Esta tornou-se uma doença de notificação obrigatória por meio da Portaria Ministerial número 542 de 22 de dezembro de 1986.

Já a sífilis em gestantes passou a constar entre as doenças e agravos de notificação obrigatória por meio da Portaria Ministerial número 33, de 14 de julho de 2005, e a sífilis adquirida em 2011, com a Portaria Ministerial número 144, de 25 de janeiro ${ }^{(5)}$. No entanto a subnotificação é frequente, como verificado em um estudo em Palmas (TO) e no Estado de São Paulo, onde por meio da busca ativa verificou se o triplo de casos identificados, em comparação com os notificados ${ }^{6}$.

Há variáveis relacionadas a efetividade da notificação os casos como a capacidade de intervenção dos serviços de saúde, capa- 
cidade de identificação dos casos por parte da equipe de saúde. Dessa forma, um baixo número de casos de SC não indica necessariamente um bom programa de controle de transmissão vertical, uma vez que há a possibilidade de subnotificação. Por outro lado, um número elevado indica falhas no processo assistencial, com oportunidades perdidas de intervenção(1).

O pré-natal tem um papel importante na redução dos desfechos perinatais negativos, ao monitorar o desenvolvimento da gravidez, diagnosticar e tratar intercorrências clínicas e obstétricas com repercussão para a saúde materna e fetal, realizar ações profiláticas específicas e reduzir a exposição da gestante e do feto a fatores de risco ${ }^{(7)}$. Cabe ressaltar que as ações de prevenção da SC, estão diretamente relacionadas aos cuidados com a gestante durante o pré-natal, como realização dos testes sorológicos para sífilis durante a gestação, o tratamento correto e oportuno da infecção diagnosticada, instituído o mais precoce, tanto da muIher, quanto do parceiro $^{(8)}$. Sendo assim, a SC é considerada, em termos epidemiológicos, um indicador da qualidade da assistência pré-natal e o tratamento adequado da gestante é o melhor método de prevenção ${ }^{(3)}$.

O Governo Federal implantou o Programa Rede Cegonha em 2011, e uma de suas estratégias é a implantação dos testes rápidos para diagnóstico da sífilis, no intuito de promover diagnóstico precoce do agravo e início do tratamento em tempo oportuno ${ }^{(9)}$. No Estado do Paraná, o Programa Rede Mãe Paranaense, também direciona a atenção às Infecções Sexualmente Transmissíveis (ISTs), em particular, a sífilis, que é apresentado também pela Rede Cegonha ${ }^{(10)}$. Todos os profissionais de saúde devem estar aptos a reconhecer as manifestações clínicas da sífilis, assim como interpretar os resultados dos exames laboratoriais, a confirmação do diagnóstico e o monitoramento da resposta ao tratamento ${ }^{(11)}$.

Ante o exposto, posta justificativa para se dedicar à pesquisa no problema enunciado, emergiu a seguinte indagação: Qual o perfil dos casos de sífilis materna e SC notificados em um Hospital de Ensino? Sendo assim, este estudo teve como objetivos co- nhecer o perfil dos casos de sífilis materna e SC notificados em um hospital de ensino.

\section{MÉTODO}

Estudo descritivo, retrospectivo e documental, com análise quantitativa, dos casos de sífilis materna e sífilis congênita notificados pelo Núcleo de Vigilância Epidemiológica Hospitalar (NVEH) de um hospital de ensino, sendo este um campo para aulas práticas de cursos técnicos, de graduação e de pós-graduação na modalidade de residência em enfermagem, medicina, fisioterapia, farmácia e odontologia.

Os dados foram coletados junto ao Sistema de Informação de Agravos de Notificação (SINAN) de todas as fichas de notificação de sífilis materna e SC notificadas no período de 2007 a 2017. Para a coleta de dados foi construído um instrumento, sendo este um roteiro, dividido em três partes, a primeira parte pertinente as características sócios demográficas maternas, a segunda relativa as características do pré-natal e a terceira parte com dados do recém-nascido. Todas as variáveis selecionadas para o estudo compõem a ficha de notifi- cação obrigatória de sífilis.

No período do estudo foram notificados 126 casos de sífilis materna e SC, destes foram excluídas 12 fichas de notificação que estavam incompletas, sendo assim, o total da amostra deste estudo foi 114 casos de sífilis materna e SC.

Os dados obtidos foram sumarizados em planilhas eletrônicas do software Microsoft Office Excel@ versão 2007 e, posteriormente, analisados por estatística descritiva, obtida pelas medidas de frequência relativa e absolutas dos casos notificados e posteriormente analisados e dispostos em quadro e tabelas.

Este estudo foi aprovado pelo Comitê de Ética e Pesquisa que envolve Seres Humanos, da Universidade Estadual do Oeste do Paraná/UNIOESTE, conforme parecer número 2.751.985 e CAAE 90600318.3.0000.0107, respeitando-se os princípios da Resolução 466/2012 da Comissão Nacional de Ética e Pesquisa Envolvendo Seres Humanos.

\section{RESULTADOS}

No período do estudo, foram notificados 114 casos de sífilis materna e SC.
Tabela 1: Característica sócio demográficas das mulheres com sifilis materna no período de 2007 a 2017. Cascavel/PR. 2018.

$$
\text { Variável }
$$

N

$\%$

\section{Faixa etária (anos)}

\begin{tabular}{lcc} 
Até 19 anos & 29 & 25,43 \\
20 a 24 anos & 39 & 34,21 \\
\hline 25 a 29 anos & 19 & 16,66 \\
\hline 0 a 34 anos & 17 & 14,92 \\
\hline Acima de 35 anos & 10 & 8,78 \\
\hline Cor da pele & & \\
\hline Branca & 85 & 74,57 \\
Preta & 2 & 1,75 \\
\hline Parda & 23 & 20,18 \\
Ignorada & 4 & 3,50 \\
\hline Escolaridade & & 2,63 \\
\hline Analfabeta & 3 & 31,58 \\
\hline Ensino Fundamental incompleto & 36 & 14,03 \\
\hline Ensino Fundamental completo & 16 & 8.78 \\
\hline Ensino Médio completo & 10 &
\end{tabular}




\begin{tabular}{lcc} 
Ensino superior completo & 2 & 1,75 \\
\hline Ignorado & 24 & 21,05 \\
\hline Área & & \\
\hline Urbana & 102 & 89,47 \\
\hline Rural & 5 & 4,39 \\
\hline Ignorado & 7 & 6,14 \\
\hline
\end{tabular}

Fonte: Banco de dados dos pesquisadores/2018.

Tabela 2: Características do pré-natal das mulheres com sifilis materna no período de 2007 a 2017. Cascavel/PR. 2018.

$$
\text { Variável } \quad \mathrm{N} \quad \%
$$

Realizou pré-natal na gestação

\begin{tabular}{lcc|}
\hline Sim & 100 & 87,71 \\
\hline Não & 10 & 8,78 \\
\hline Ignorado & 4 & 3,50 \\
\hline Diagnóstico da sífilis materna & & \\
\hline Durante o pré-natal & 75 & 65,79 \\
\hline No momento do parto/curetagem & 29 & 25,43 \\
\hline Não realizado & 9 & 7,90 \\
\hline Ignorado & 1 & 0,88 \\
\hline
\end{tabular}

Teste não treponêmico no parto/ curetagem

\begin{tabular}{|lcc|}
\hline Reagente & 99 & 86,84 \\
\hline Não reagente & 6 & 5,26 \\
\hline Não realizado & 7 & 6,14 \\
\hline Ignorado & 2 & 1,75 \\
\hline Esquema de tratamento & & \\
\hline Adequado & 4 & 3,50 \\
\hline Inadequado & 66 & 57,90 \\
\hline Não realizado & 41 & 35,96 \\
\hline Ignorado & 3 & 2,63
\end{tabular}

Parceiro tratado concomitante com a mulher

$\begin{array}{lcc}\text { Sim } & 21 & 18,42 \\ \text { Não } & 86 & 75,43 \\ \text { Ignorado } & 7 & 6,14\end{array}$

Fonte: Banco de dados dos pesquisadores/2018

TABELA 3: Distribuição anual da ocorrência de siffilis congênita no período de 2007 a 2017. Cascavel/PR. 2018.

$\begin{array}{llll} & \text { Ano } & \text { N } & \% \\ 2007 & & 3 & 2,63 \\ 2008 & 4 & 3,50 \\ 2009 & 2 & 1,75\end{array}$

Quanto a área de residência, foi encontrado 102 casos de moradores da área urbana $(89,47 \%)$, área rural, cinco casos $(4,38 \%)$ e ignorado sete casos $(6,14 \%)$. A faixa etária com maior incidência da sífilis materna foi entre 20 a 24 anos com 39 casos (34.21\%), a cor predominante das mulheres com sífilis foi a branca com 75 casos $(65,78 \%)$ e a escolaridade predominante foi o ensino fundamental incompleto, com 36 casos $(31,57 \%)$. Conforme demonstrado na tabela I.

A tabela I mostra as características do pré-natal, do total da amostra, 100 $(87,71 \%)$ das mulheres com diagnóstico de sífilis, realizaram pré-natal, não sendo possível quantificar o número de consultas, bem como em qual trimestre iniciou o pré-natal. Quanto ao diagnóstico da sífilis materna, 75 mulheres $(65,78 \%)$ tiveram o diagnóstico no pré-natal, 29 (25,43\%) tiveram o diagnóstico no momento do parto ou da curetagem, 99 mulheres $(86,84 \%)$ tiveram teste não treponêmico reagente, somente $4(3,50 \%)$ foram adequadamente tratadas e 66 mulheres $(57,89 \%)$ tiveram tratamento inadequado para sífilis.

Quanto ao tratamento do parceiro concomitante ao das mulheres foi encontrado $86(75,43 \%)$ casos não tratados e somente $21(18,42 \%)$ tratados como evidenciado na tabela II.

A tabela III mostra a distribuição anual dos casos de sífilis materna e congênita. A partir do ano de 2012 ocorreu um aumento de casos, com maior concentração no ano de 2017.

A tabela IV apresenta as características do recém nato com sífilis congênita. Quanto ao sexo, 58 (50,87\%) dos casos eram masculinos e $53(46,49 \%)$ femininos, com predomínio da cor branca 93 $(81,57 \%), 83(72,80 \%)$ dos recém natos tiveram o teste não treponêmico (sangue periférico) reagente e 16 recém natos $(14,03 \%)$ o teste não foi realizado. Quanto ao esquema de tratamento $54(47,36 \%)$ foram tratados com Penicilina 100.000 a $150.000 \mathrm{Ul} / \mathrm{kg} / \mathrm{dia} / 10$ dias. Relativo a evolução do caso, 96 (84,21\%) dos recém natos com diagnóstico positivo para sífilis, estavam vivos no momento da alta. 


\begin{tabular}{|lcc|}
\hline 2010 & 1 & 0,88 \\
\hline 2011 & 5 & 4,39 \\
\hline 2012 & 17 & 14,91 \\
\hline 2013 & 13 & 11,40 \\
\hline 2014 & 10 & 8,78 \\
\hline 2015 & 17 & 14,92 \\
\hline 2016 & 17 & 14,92 \\
\hline 2017 & 25 & 21,92 \\
\hline Total & 114 & 100 \\
\hline Fonte: Banco de dados dos pesquisadores/2018. & & \\
\hline
\end{tabular}

\section{DISCUSSÃO}

A maior concentração de sífilis materna foi para a faixa etária de 20 a 24 anos, muIheres jovens de cor branca, com ensino fundamental incompleto e moradoras da área urbana. Os dados do presente estudo corroboram a favor de que a sífilis tem importante ocorrência, assim como outras ISTs, devendo ser fundamental a prevenção para toda a população. A dificuldade de prevenção da transmissão permanece no entrave do diagnóstico e do tratamento precoce e adequado ${ }^{(13)}$.

Este estudo evidenciou que a maioria Tabela 4: Características do recém nato com sífilis congênita no período de 2007 a

\section{Cascavel/PR. 2018.}

$$
\text { Ano N } \quad \%
$$

$\mathrm{N} \quad \%$

\section{Sexo}

\begin{tabular}{lcc}
\hline Masculino & 58 & 50,87 \\
\hline Feminino & 53 & 46,50 \\
\hline Ignorado & 3 & 2,63 \\
\hline Cor & & \\
\hline Branca & 93 & 81,58 \\
\hline Negro & 2 & 1,75 \\
\hline Pardo & 12 & 10,52 \\
\hline Ignorado & 7 & 6,14
\end{tabular}

\section{Teste não treponêmico - Sangue periférico}

\begin{tabular}{lcc} 
Reagente & 83 & 72,80 \\
\hline Não reagente & 12 & 10,53 \\
\hline Não realizado & 16 & 14,03 \\
\hline Ignorado & 3 & 2,63 \\
\hline Esquema de tratamento & & \\
\hline Pen G Cristalina 100.000 a 150.000 UI/kg/dia/10 dias & 54 & 47,37 \\
\hline Pen G Procaína 50.000 UI/kg/dia/10 dias & 14 & 12,29 \\
\hline Pen G Benzatina 50.000 UI/kg/dia & 7 & 6,14 \\
\hline Outro & 16 & 14,03 \\
\hline Não realizado & 15 & 13,15 \\
\hline Ignorado & 8 & 7,01 \\
\hline Evolução & & 84,21 \\
\hline Vivo & 96 & 4,38 \\
\hline Óbito por sífilis congênita & 5 & 0,88 \\
\hline Aborto & 1 & 7,90 \\
\hline Natimorto & 9 & 2,63 \\
\hline Ignorado & 3 & \\
\hline Forto Ban & & \\
\hline
\end{tabular}

Fonte: Banco de dados dos pesquisadores/2018 das gestantes realizou pré natal, tiveram diagnóstico da sífilis durante o pré-natal, assim como mulheres com diagnóstico da sífilis no momento do parto ou da curetagem. Outros estudos igualmente apontaram tais resultados, com a realização do pré natal conforme preconizado, mas, com a não realização dos exames de rastreamento, evidenciando a dificuldade do diagnóstico e tratamento ${ }^{14)}$.

Considerando o tratamento, 66 (57,90\%) mulheres foram tratadas inadequadamente e em 86 (75,43\%) dos casos o parceiro não foi tratado concomitantemente. O tratamento é realizado com penicilina, utilizando dose da medicação conforme estágio da doença e o parceiro sexual deve ser devidamente medicado concomitantemente ${ }^{(11)}$.

O tratamento inadequado é extremamente comum, como verificado neste trabaIho e por vários outros estudos e sua principal causa é observada em relação ao tratamento dos parceiros das gestantes ${ }^{(8)}$. Um estudo realizado investigou o motivo do não tratamento dos parceiros e verificou que eles são informados da doença e simplesmente não realizam o tratamento, questionando se a questão cultural do adoecer no homem ${ }^{(15)}$.

Conforme a distribuição dos casos por ano, temos que a partir do ano de 2012 houve um aumento no número dos casos, porém não é possível afirmar se ocorreu melhora nas notificações ou a implantação de políticas voltadas para o controle e a eliminação da sífilis. Quanto às características do recém nato com sífilis congênita não houve diferença significativo entre a ocorrência por sexo, predominando a cor branca, esta variável pode ser 
justificada em função da localidade na qual ocorreu a pesquisa colonizada predominantemente por alemães e italianos.

A maioria dos recém natos tiveram o teste não treponêmico (sangue periférico) reagente. Quanto ao esquema de tratamento 54 (47,37\%) foram tratados com Penicilina G Cristalina, intravenosa por 10 dias $^{(11)}$. Relativo a evolução do caso, 96 (84,21\%) recém natos com diagnóstico positivo para sífilis tiveram boa evolução, tendo alta hospitalar e 5 (4,38\%) indo a óbito devido a patologia.

Todos os recém-nascidos de gestantes com sífilis devem ser investigados para SC. Somente por meio de um esforço unificado, com assistência pré natal de qualidade, exa $\neg$ mes laboratoriais em tempo hábil, tratamento do casal e sensibilização de todos envolvidos, será possível alcançar o objetivo almejado de controle dessa infecção ${ }^{(16-17)}$. Devendo se utilizar a Estratégia Saúde da Família como forma de aproximação da população, conhecendo que é uma das principais portas de entrada para os serviços de saúde, e dos agentes comu $\neg$ nitários de saúde para busca ativa às gestantes, assim investindo na educação continuada ${ }^{(18)}$. O tratamento adequado da sífilis na gestação é uma maneira efetiva e acessível de impedir e prevenir a transmissão vertical e, por consequência, a infecção congênita ${ }^{(14)}$.

Este estudo teve como limitação, o não conhecimento da importância e aplicabilidade das notificações, fazendo com que muitas doenças e agravos não se tornem conhecidos e investigados. Assim, a eficiência da notificação depende da contínua sensibilização dos profissionais de saúde, para que reconheçam a importância dessa informação, com consequente melhoria da cobertura e da qualidade dos dados disponíveis ${ }^{(19)}$.

\section{CONCLUSÃO}

A sífilis congênita continua sendo um problema de saúde pública. Embora o diagnóstico e o tratamento são de fácil acesso e sem custo para o paciente, disponibilizado integralmente pelo Sistema Único de Saúde, esse agravo ainda é um desafio para a redução dos casos.

A sífilis em gestantes e congênita permanece como uma doença a ser prevenida e controlada, em que se busca o controle da doença. Os resultados também evidenciam aumento no número de casos em gestantes e a persistência da transmissão vertical, sinalizando, assim, para a necessidade de adesão por parte dos profissionais de políticas de controle da sífilis, bem como melhoria nas notificações, na sensibilização e divulgação das informações, deste agravo, quanto às formas de promoção e prevenção.

É necessário um olhar diferenciado por parte dos gestores, incentivando a utilização das políticas e normas de rastreamento sistemático e terapêutica adequada, visto que existem políticas públicas bem definidas necessitando apenas que sejam observadas e praticadas de forma a reduzir essa doença. Pode-se concluir ser de fundamental importância a análise dos casos de sífilis, bem como os fatores envolvidos, para o desenvolvimento de ações de prevenção e controle da doença. 1

\section{Referências}

1. Silva MAM, Sousa AJC, Albulquerque ES, Moreira ACA, Martins KMC. Feelings of pregnant women diagnosed with syphilis. Rev Enferm UFPI. 2015;4(2):84-91. 2. Cavalcante AES, Silva MAM, Rodrigues ARM, Netto JJM, Moreira ACA, Goyanna NF. Diagnóstico e Tratamento da Sífilis: uma Investigação com Mulheres Assistidas na Atenção Básica em Sobral, Ceará. Jornal Bras. Doenças Sexualmente Transmissiveis, p. 239, 2012. Disponível em: <http://www.dst.uff.br/revista24-4-2012/ JBDST2012-24-4.pdf\#page=16> Acesso: 06 dez de 2018.

3. Lafetá KRG, Herćilio, Martelli Júnior H, Silveira MF, Paranaíba LMR. Sífilis materna e congênita, subnotificação e difícil controle. Rev. Bras. Epidemiologia. Jan-Mar 2016; 19(1): 63-74.

4. Ministério da Saúde (BR). Sífilis: estratégias para diagnóstico no Brasil. Coordenação de Doenças Sexualmente Transmissíveis e Aids. Brasília/DF, 2010. Disponivel em: http://www.aids.gov.br/sites/default/files/anexos/page/2012/50768/ manual_sifilis_miolo_pdf_53444.pdf $>$. Acesso em: 07dez de 2018.

5. Ministério da Saúde (BR). Aids e Hepatites virais. Protocolo clínico e diretrizes terapêuticas para prevenção da transmissão vertical de HIV, Sífilis e Hepatites Virais. Secretaria de Vigilância em Saúde Departamento de DST. ${ }^{1}{ }^{a}$ edição - Brasilia/DF, 2015. Disponivel em: <http://www.aids.gov.br/sites/default/files/anexos/ publicacao/2015/58572/pcdt_transm_vertical_091215_pdf_12930.pdf $>$ Acesso em: 07dez de 2018.

6. São Paulo. Serviço de Vigilância Epidemiológica. Coordenação de DST/Aids SP. Informe Técnico Institucional. Secretaria de Estado de Saúde SES SP. Sífilis congênita e sífilis na gestação. Rev. Saúde Pública 2008; 42(4): 76872.

7.Schust ABH, Suezawa YD, Elias PG, Barbosa AA, Blanco BA. Rev. Saúde em Foco. Edição número 9.2017.

8. Lima MG, Ribeiro GS, Santos RFR, Barbosa GJA. Incidência e fatores de risco para sífilis congênita em Belo Horizonte, Minas Gerais, 2001 2008. Ciênc. Saúde Coletiva 2013; 18(2): 499506

9. Ministério da Saúde (BR). Programa Rede Cegonha. Brasília DF, 2012.

10. Secretaria de Estado da Saúde do Paraná (SESA-PR). Programa Rede Mãe Paranaense. Linha guia. SESA-PR: Curitiba PR, 2017.
11. Ministério da Saúde (BR). Aids e Hepatites virais. Protocolo clínico e diretrizes terapêuticas para prevenção da transmissão vertical de HIV, Sifilis e Hepatites Virais. Secretaria de Vigilância em Saúde Departamento de DST. 1 a edição - Brasília - DF, 2015. Disponível em: <http://www.aids.gov.br/sites/default/files/anexos/ publicacao/2015/58572/pcdt_transm_vertical_091215_pdf_12930.pdf >Acesso em: 06dez de 2018.

12. Brasil. Comissão Nacional de Ética em Pesquisa. Resolução 466 de 12 de dezembro de 2012, dispõe sobre normas e diretrizes regulamentadoras de pesquisa envolvendo seres humanos. Brasilia, 2012.

13. Nonato SM, Melo APS, Guimarães MDC. Sífilis na gestação e fatores associados à sífilis congênita em Belo Horizonte/MG, 2010-2013. Rev. Epidemiol. Serv. Saúde, vol. 24, Brasilia - DF, 2015. Disponível em: <http://www.scielo.br/scielo. php?script=sci_arttext\&pid=S2237-6222015000400681\&lng=pt\&nrm=iso $>$. Acesso em: 07dez de 2018.

14. Campos ALA, Araújo MAL, Melo SP, Gonçalves MLC. Epidemiologia da sífilis gestacional em Fortaleza, Ceará, Brasil: um agravo sem controle. Cad. Saúde Pública 2010; 26(9): 174755.

15. Campos ALA, Araújo MAL, Melo SP, Andrade RFV, Gonçalves MLC. Sífilis em parturiente: aspectos relacionados ao parceiro sexual. Rev. Bras. Ginecol. Obstet. 2012; 34(9): 397402

16. Magalhães DMS, Kawaguchi IAL, Dias A, Calderon IMP. A sifilis na gestação e a sua influência na morbimortalidade materno infantil. Comum. Ciênc. Saúde 2011; 1(22): 4354.

17. Kupek E, Oliveira JF. Transmissão vertical do HIV, da sífilis e da hepatite B no município de maior incidência de AIDS no Brasil: um estudo populacional no período de 2002 a 2007. Rev. Bras. de Epidemiologia 2012; 15(3): 47887.

18. Costa CC, Freitas LV, Sousa DMN, Oliveira LL, Chagas ACMA, Lopes MVO, et al. Sífilis congênita no Ceará: análise epidemiológica de uma década. Rev. Esc. Enferm. USP 2013; 47(1): 15259.

19. Brasil. Fundação Nacional de Saúde. Guia de Vigilância Epidemiológica/Fundação Nacional de Saúde. 5. ed. Brasília: FUNASA, 2002. 842p. 